\title{
Inhibition of mTOR signaling pathway by aqueous extract of Siberian ginseng
}

\author{
Boo Hyeong Byun ${ }^{1}$, Tae Hwan $\mathrm{Cho}^{2}$, Kyeong Mee Park ${ }^{3}$ \\ ${ }^{1}$ Department of Oriental Medicine, Daegu Haany University \\ ${ }^{2}$ Cho Oriental Clinic \\ ${ }^{3}$ Inno Oriental Clinic
}

\begin{abstract}
Objectives: This study evaluated the effect of aqueous extract from roots of Siberian ginseng on mTORC1 pathway. Methods: mTORC1 activity was measured by the phosphorylation status of p70 S6 kinase (S6K) in HeLa cells as well as the brain, liver and muscle tissues in diabetic $\mathrm{db} / \mathrm{db}$ mice. Autophagy induction after the treatment of Siberian ginseng extract was evaluated by monitoring the conversion of cytoplasmic LC3I into lipidated LC3II in cultured human HeLa GFP-LC3 cells. Cell cycle analysis was performed in HeLa cells treated with Siberian ginseng using flow cytometry.

Results: Among $>2,800$ plant products used for oriental medicine, Siberian ginseng was found to inhibit mTORC1 to phosphorylate S6 kinsase (S6K) in HeLa cells as well as the brain, liver and muscle tissues in diabetic db/db mice. Siberian ginseng-mediated mTORC1 activity was reversible unlike the prolonged suppression of mTORC1 by rapamycin when HeLa cells were grown in fresh media after the removal of the inhibitors. Siberian ginseng extract at concentrations to inhibit mTORC1 was not overly cytotoxic in cultured HeLa cells whereas rapamycin was obviously cytotoxic. The conversion of cytoplasmic LCI into lipidated LCII was increased by fivefold in HeLa GFP-LC3 cells treated with Siberian ginseng extract. Progression of cell cycle was attenuated at G2/M phase by the treatment of Siberian ginseng extract.

Conclusions: These results suggest that the aqueous extract of Siberian ginseng possibly plays a good therapeutic role in various diseases involving mTORC1 signaling.
\end{abstract}

$\overline{\text { Key Words }}$ : Siberian ginseng, Acanthopanacis senticosi Radix, autophagy, cell cycle, mTORC1, S6K.

\section{Introduction}

Mammalian target of rapamycin (mTOR) signaling pathway has been regarded as a central controller of cell growth, cell size, cell shape and cell proliferation in mammalian cells. mTOR is a Ser/Thr kinase that belongs to the phosphatidylinositol kinase-related protein kinase family ${ }^{1)}$. mTOR exists in two complexes, mTORC1 and mTORC2. While mTORC1 is a rapamycin-sensitive protein kinase which regulates protein synthesis and cell growth, mTORC2 is a rapamycin-resistant protein kinase which promotes cell survival and cytoskeleton organization ${ }^{2-4)}$. mTORC1 activates cellular processes by phosphorylating two downstream effectors, ribosome S6 kinases (S6K) and eukaryotic initiation factor 4E-binding proteins $(4 \mathrm{E}-\mathrm{BPs})^{5,6}$. Both mTORC1 and $\mathrm{S} 6 \mathrm{~K}$ are activated by insulin through the insulin receptor/insulin receptor substrate/phosphatidyinositol 3-kinase/Akt (PI3-kinase/Akt) pathway $^{7)}$.

\footnotetext{
- Received : 30 March 2017

- Revised : 15 June 2017

- Accepted : 15 June 2017

- Correspondence to : Kyeong Mee Park

Inno Oriental Clinic, Gwanpyeong-Dong, Yusung-Gu, Daejon 34014, South Korea

Tel : +82-42-671-9933, Fax : +82-42-671-9969, E-mail : kmpark02@empas.com
} 
Studies have demonstrated that mTORC1 activity upregulates anabolic cellular processes including protein synthesis and ribosome biogenesis, but downregulates catabolic processes such as autophagy ${ }^{1)}$. As mTORC1 signaling is centered at the interphase between catabolic and anabolic processes, mTORC1 is implicated in diseases associated with dysregulation of cell growth and failure of maintaining homeostasis. Activation of mTORC1 pathway has been known to be involved in various pathological conditions including cancer, obesity and type II diabetes ${ }^{8,9)}$. Recent findings have also presented evidence that the inhibition of mTORC1 signaling pathway can extend lifespan in various organisms including mice ${ }^{10,11)}$.

Rapamycin has been shown to prevent cell cycle progression from $\mathrm{G} 1$ to $\mathrm{S}$ phase in mammalian cells ${ }^{12}$. Rapamycin and its derivatives, which act as specific allosteric inhibitors of mTORC1 kinase ${ }^{13)}$, have been developed as anticancer agents in various preclinical models ${ }^{14)}$, and clinically used as an immunosuppressant for organ transplantation ${ }^{15,16)}$. Despite of a strong therapeutic potential, rapamycin has some unwanted side effects such as mTOR inhibitor-associated lymphedema ${ }^{17)}$, stomatitis ${ }^{18)}$ and azoospermia ${ }^{19)}$.

Therefore, researchers have tried to find out efficient inhibitors of mTOR signaling pathways without adverse side effects. In search of naturally occurring plant products that can modulate mTORC1 activity, a large collection of $>2,800$ natural plant products used in folk medicine for pharmacological applications was screened. Among 2,800 plant products, Siberian ginseng was found to inhibit to inhibit mTOR/S6K activity, induce autophagy and arrest cell cycle at $\mathrm{G} 2 / \mathrm{M}$ phase in cultured human cells. We used obese diabetic $\mathrm{db} / \mathrm{db}$ mice to investigate whether the aqueous extract of Siberian ginseng suppresses the phosphorylation of $\mathrm{S} 6 \mathrm{~K}$ in the brain, liver and muscle tissues.

In this report, we launch revealing out the mechanism of Siberian ginseng, which has been traditionally used in Korean medicine for a wide range of health benefits ${ }^{20)}$, on the intervention in mTOR signaling.

\section{Material and Methods}

\section{Reagents and antibodies}

Mouse monoclonal antibody for S6K and Anti-GFP mouse antibody were from Santa Cruz Biotechnology (Santa Cruz, CA, USA). Mouse antibody against phosphorylated S6K (Thr 389) was from Cell Signaling Technology (Boston, MA, USA). Rapamycin and rabbit antibodies for phosphorylated Akt at Thr 308 and Ser 473 were also from Cell Signaling Technology. Anti-a-tubulin antibody (T5168) was purchased from Sigma Chemical Co. (St. Louis, MO, USA).

\section{Preparation of an aqueous extract from roots of Siberian ginseng}

Dried Siberian ginseng roots (Acanthopanacis senticosi Radix) cultivated in Youngcheon Province, South Korea were obtained from National Medicine Research Center of Korean Instititute of Bioscience and Technology, Daejon, South Korea. The plant was identified by a botanist from the Institute of Traditional Medicine and Bioscience, Daejon University, Daejon, South Korea. Roots of Siberian ginseng $(600 \mathrm{~g})$ were pulverized, extensively washed with water and dried at $40^{\circ} \mathrm{C}$ in a hot air oven. The plant material was homogenized in $2 \mathrm{~L}$ of hot boiling water for $2 \mathrm{~h}$ and allowed to stand for $24 \mathrm{~h}$. After filtration, the filtrate was lyophilized. The lyophilized extract $(\sim 50 \mathrm{~g})$ was dissolved in DMSO and used to evaluate its effect on mTORC1 signaling in both cultured cells and animal model.

\section{Cell culture and viability assay}

HeLa cells were cultured in Dulbecco's Modified 
Eagle's medium (DMEM) supplemented with $10 \%$ (v/v) fetal bovine serum (Gibco, Grand island, NY, USA) and maintained at $37^{\circ} \mathrm{C}$ in $5 \%(\mathrm{v} / \mathrm{v}) \mathrm{CO}_{2}$ under a humidified atmosphere. Viability of HeLa cells after treatment with rapamycin $(50-200 \mathrm{nM})$ or Siberian ginseng $(50-100 \mu \mathrm{g} / \mathrm{ml})$ was determined by trypan blue dye exclusion as described elsewhere ${ }^{21)}$.

\section{Experimental mice}

C57BLKS/J-m+/Lepr $\mathrm{db}(\mathrm{db} / \mathrm{db})$ mice with 14 week-old age were purchased from Japan SLC, Inc and acclimated to the experimental condition of Korea Advanced Institute of Science and Technology (KAIST) for 1 week. Experiments using this mouse strain were compliant with the guideline of Institutional Animal Care and Use Committee (IACUC) at KAIST. Diabetic obese $\mathrm{db} / \mathrm{db}$ mice were fed a normal diet AIN-76A, (Research Diets, USA) composed of $20.3 \% \quad(\mathrm{~g} / \mathrm{g})$ protein, $66 \% \quad(\mathrm{~g} / \mathrm{g})$ carbohydrate, $5 \%(\mathrm{~g} / \mathrm{g})$ fat and $8.7 \%(\mathrm{~g} / \mathrm{g})$ essential ingredients under ad libitum conditions. Siberian ginseng extract $(100 \mu \mathrm{g} / \mathrm{g}$ body weight/day) was intraperitoneally injected to 15 week-old mice $(n=3$ female and 3 male mice) for a week. After $24 \mathrm{~h}$ of the seventh injection, mice were sacrificed. All the mice were starved for $16 \mathrm{~h}$ before sacrifice. For analysis of mTORC1 activity, tissue samples were prepared from the sacrificed mice for immunoblotting.

\section{Induction of autophagy}

Autophagy induction after the treatment of Siberian ginseng extract was evaluated by monitoring the conversion of cytoplasmic LC3I into lipidated LC3II ${ }^{22}$ in cultured human HeLa GFP-LC3 cells. GFP-LC3 HeLa cells were established as previously described ${ }^{23)}$. The induction of autophagy by the plant extract was quantified by measuring the percentage of GFP-LC3 positive HeLa GFP-LC3 cells with punctuate dots using fluorescence microscopy.

\section{Cell cycle analysis}

Cell cycle was analyzed by flow cytometry. HeLa cells treated with Siberian ginseng extract were fixed in $1 \%$ formaldehyde PBS followed by ice-cold $70 \%$ ethanol, and incubated on ice for 30 mins with thorough mixing. Fixed cells were then treated with RNaseI and stained with propidium iodide for fluorescence-activated cell sorter (FACS) analysis of DNA contents ${ }^{24)}$.

\section{Results and Discussion}

\section{Inhibition of mTORC1 activity by Siberian ginseng extract in cultured HeLa cells}

An aqueous extract from roots of Siberian ginseng inhibited ability of mTORC1 to phosphorylate its substrate protein S6K in HeLa cells (Fig. 1A). Due to the presence of mTORC1-dependent negative feedback loop, inactivation of mTORC1 results in the increases in the phosphorylation of insulin receptor substrate-1 (IRS-1) and mTORC2 ${ }^{9}$. Rapamycin, for example, inhibits the phosphorylation of S6 kinase due to the inhibition of mTORC1, but stimulates the phosphorylation of $\mathrm{Akt}^{25)}$. Akt is either phosphorylated by PI3-kinase at Thr 308 or phosphorylated at Ser 473 by mTORC2 $2^{25-27)}$. Since IRS-1/PI3K pathway is negatively regulated by the mTORC1-dependent S6K phosphorylation, mTORC1 inhibition primes the activation of phosphorylation of Akt at Thr 308 by IRS-1/PI3K signal pathway. Aside from Akt phosphorylation at $\mathrm{Thr} 308$, inhibition of S6K by mTORC1 inactivation also upregulates mTORC2 through the inactivation of phosphorylation of Rictor component in mTORC2. Therefore, the inhibition of S6K activity through mTORC1 inactivation enhances the activity of mTORC2, which in turn activates Akt through its phosphorylation at Ser $473^{28,29)}$.

In our study, data in Fig. 1B revealed that mTORC1 inhibition by Siberian ginseng extract 
might also elicit the increased Akt phosphorylation at Thr 308 through the activation of IRS-1/PI3K pathway as well as the increased Akt phosphorylation at Ser 473 through the activation of mTORC2 pathway. These results evidenced the presence of the negative feedback loops that induce mTORC1 inactivation mediated by the upregulation of IRS-1/ PI3K or mTORC2. The increased phosphorylation of Akt after the treatment with Siberian ginseng extract

A

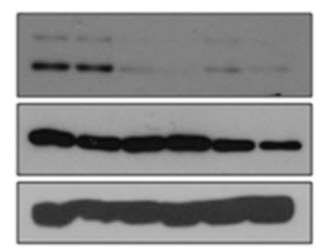

$\mathrm{ps} 6 \mathrm{~K}$

S6K

Tubulin paradoxically advocated the substantial inhibition of mTORC1 by Siberian ginseng in cultured human cells. The underlying mechanism by which Siberian ginseng extract inhibits mTORC1 remains unknown at present. The increased phosphorylation of Akt following by the inhibition of mTORC1 (Fig. 1B) at least disprove that Siberian ginseng extract inhibits mTORC1 by inhibiting Akt, an upstream signaling kinase that activates mTORC1. The activation of Akt

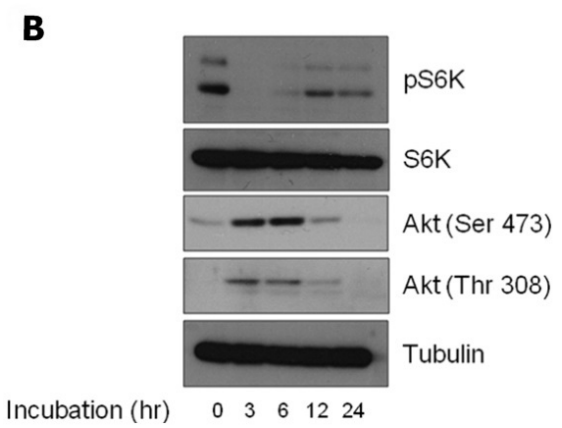

C
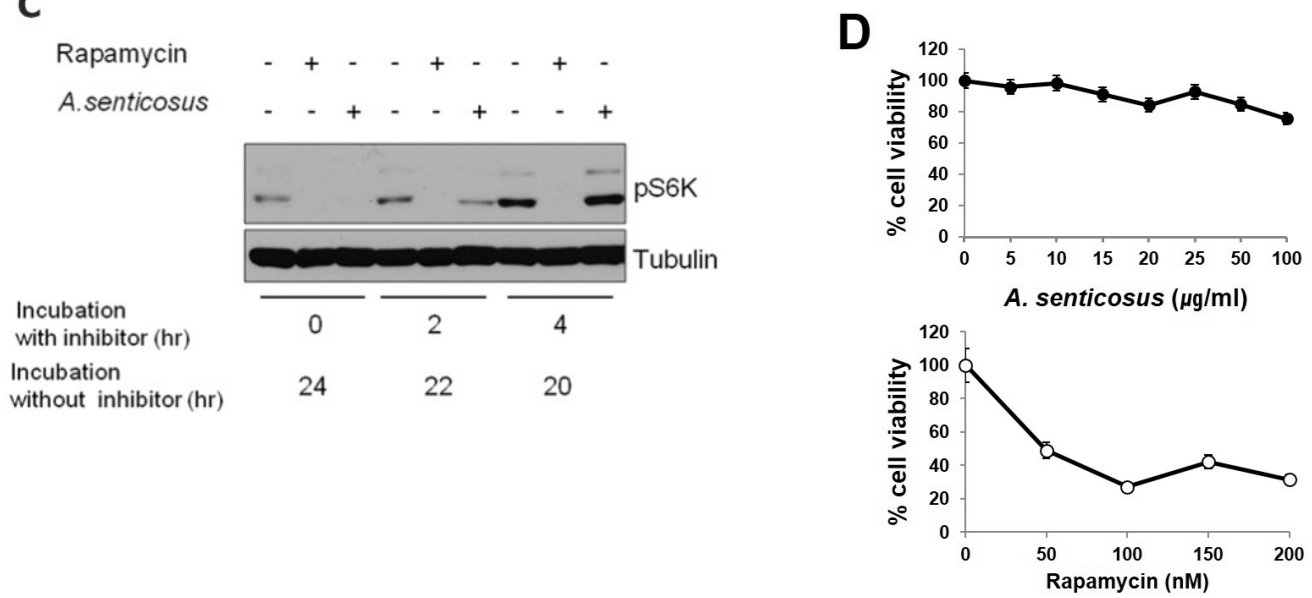

Fig. 1. mTORC1 inhibition in cultured HeLa cells treated with an aqueous extract from roots of Siberian ginseng (A. senticosus). (A) Inactivation of mTORC1 in HeLa cells treated with A. senticosus extract was analyzed by immunoblotting to examine the phosphorylation status of S6K.

(B) mTORC1 inhibition by A. senticosus extract $(100 \mathrm{\mu g} / \mathrm{ml})$ for the indicated amount of time induces Akt activation in HeLa cells. Cellular levels of S6K, phosphorylated S6K, phosphorylated Akt at Ser 473 and phosphorylated Akt at Thr 308 were measured by immunoblotting using respective antibody. Tubulin level was used as a loading control.

(C) Inhibition of mTORC1 by A. senticosus extract is a reversible process whereas inhibition of mTORC1 by rapamycin is an irreversible process. HeLa cells were treated with A. senticosus extract $(50 \mathrm{\mu g} / \mathrm{mll})$ or rapamycin $(100 \mathrm{nM})$ for 0,2 or $4 \mathrm{~h}$. Cells were then washed with fresh media and post-incubated in media without inhibitors for indicated amount of time.

(D) Cytotoxicity of A. senticosus extract. HeLa cells were treated with concentrations of A. senticosus extract for $24 \mathrm{~h}$ (upper). HeLa cells were treated with concentrations of rapamycin for $24 \mathrm{~h}$ (lower). Cells were stained with $0.4 \%$ trypan blue. Total number of cells, stained and unstained, was counted. Percentages of unstained viable cells were presented. Values represent mean $\pm S D$ ( $n \geq 3$ experiments). 
after the treatment of Siberian ginseng extract also implies that mechanistic base for the inhibition of mTORC1 by Siberian ginseng is similar as that of mTORC1 inhibition by rapamycin.

However, the data in Fig. 1C disclose that inhibition of mTORC1 activity by Siberian ginseng extract is a reversible process. Unlike the prolonged suppression of rapamycin-mediated mTORC1 activity in HeLa cells grown in fresh media after the removal of rapamycin, mTORC1 inhibition by Siberian ginseng was easily recovered by growing cells in the absence of the inhibitor. The cytotoxic activity of Siberian ginseng extract was also apparently different from that of rapamycin. Siberian ginseng extract at concentrations to inhibit mTORC1 in cultured HeLa cells was not overly cytotoxic as was shown in Fig. 1D.

\section{2. mTORC1 inhibition by Siberian ginseng extract in animal model}

The effect of Siberian ginseng extract that inhibits mTORC1 was investigated in diabetic obese $\mathrm{db} / \mathrm{db}$ mice. The administration scheme of Siberian ginseng was determined from the preliminary experiment which adopted $10-100 \mu \mathrm{g} / \mathrm{g}$ body weight/day with 1-7 day protocol. In the diabetic $\mathrm{db} / \mathrm{db}$ mice in which mTORC1 activity is highly activated ${ }^{30)}$, Siberian ginseng extract $(100 \mu \mathrm{g} / \mathrm{g}$ body weight/day) intraperitoneally administered for a week induced a severe downregulation of phosphorylated S6K in tissues of brain, liver and muscle (Fig. 2).

Yet, there is no in vivo experimental evidence with anti-mTOR activity of Siberian ginseng. Supported by this result, there might be an ample probability that the oral administration of Siberian ginseng could treat and/or prevent nutrient-dependent obesity and insulin resistance in vivo.

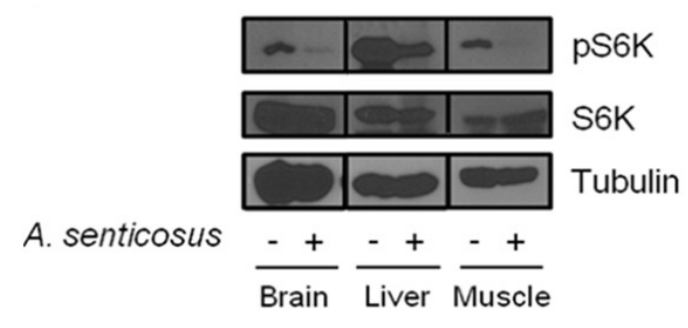

Fig. 2. Inhibition of mTORC1 in tissues of animal model treated with Siberian ginseng (A. senticosus) extract. Diabetic $\mathrm{db} / \mathrm{db}$ mice of 15 weeks of age were intraperitoneally injected with aqueous root extract of $A$. senticosus (100 $\mu \mathrm{g} / \mathrm{g}$ body weight/day) for a week ( $\mathrm{n}=3$ female and 3 male mice). Tissue homogenates from experimental mice were analyzed by immunoblotting to examine phosphorylation status of S6K and phosphorylated S6K.

\section{Autophagy induction in HeLa GFP-LC3 cells treated with Siberian ginseng extract}

mTORC1 has been well documented as a negative regulator of autophagic pathway ${ }^{7,31)}$. Autophagy is considered as a protection process which enables cells to survive adverse conditions like nutrient limitation, infections, toxins and other types of cell stress. Autophagy induction was assessed and quantified in established HeLa GFP-LC3 cells. In response to the treatment of Siberian ginseng extract, LC3I was converted into LC3II, a known hallmark of autophagy ${ }^{32)}$, in HeLa GFP-LC3 cells (Fig. 3A). The accumulation of GFP-LC3 in the autophagosomes in HeLa GFP-LC3 cells stably expressing LC3 was imaged by fluorescent microscopy (Fig. 3B) and quantified (Fig. 3C). Treatment of HeLa GFP-LC3 cells with $10 \mu \mathrm{g} / \mathrm{ml}$ of Siberian ginseng extract for 24 $\mathrm{h}$ increased the number of cells with GFP-LC3 punctate dots by approximately fivefold.

Because autophagy is a catabolic pathway that degrades bulk cytosol in the lysosomal compartments enabling amino acids and fatty acids to be recycled, attenuation in the progression of cell cycle is very plausible. 


\section{Accumulation of G2/M cell cycle by Siberian ginseng extract}

In eukaryotic cells, cell growth and cell proliferation occurs only during certain phases of cell cycle. Cell growth and cell proliferation require accumulation of cellular proteins. As cellular processes like cell growth are directly linked with mTORC1 function that integrates signals for protein synthesis, mTORC1 signaling pathway should be functionally linked with cell cycling. mTORC1 signaling pathway is wired to various proteins involved in cell cycling. These include p21 and p27 CDK inhibitor proteins, CDK4 and cyclinD1, D3, E and $\mathrm{A}^{33)}$. While rapamycin inhibits growth factor signaling and induces G1 arrest in many cell types ${ }^{12)}$, treatment of Siberian ginseng extract induces G2 arrest in HeLa cells (Fig. 4). This finding may warrant a further study on a potential use of Siberian ginseng as a chemopreventive agent with less side effects than rapamycin-based therapeutic approach.
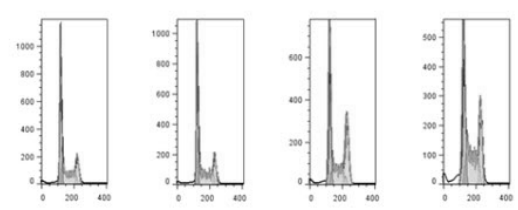

Incubation (hr) 0

Go/G1 phase (\%) $\quad 48.63$

G2/M phase (\%) 14.71

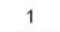

45.41

14.01

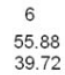

12 46.66 22.19

Fig. 4. Cell cycle arrest at G2/M phase by Siberian ginseng (A. senticosus) extract treatment. HeLa cells were treated with A. senticosus extract $(50 \mu \mathrm{g} / \mathrm{ml})$ for the stated period of time. Cells were fixed with 70\% ethanol and stained with propidium iodide (PI). Cell cycle was analyzed by flow cytometry. Percentages of G0/G1 and G2/M phases of cell cycle were presented.

\section{Acknowledgement}

We appreciate deeply Dr. Jong Soon Lim, Daejon University for supply and confirmation of Siberian ginseng. This work was in part supported by grant
A

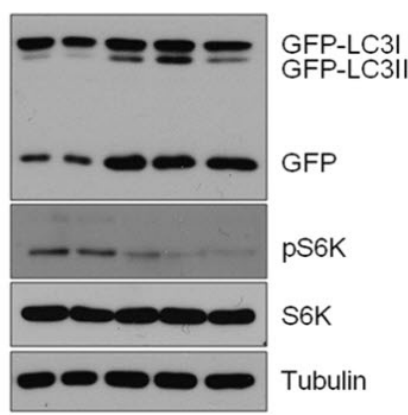

A. senticosus $(\mu \mathrm{g} / \mathrm{ml}) \quad 0 \quad 1 \quad 10 \quad 20 \quad 50$

B

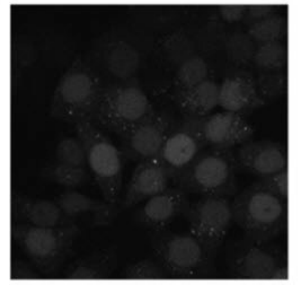

Control

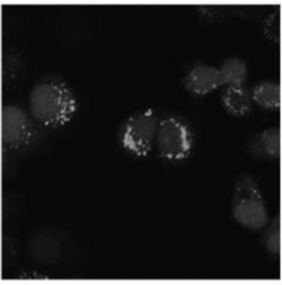

A. senticosus $(50 \mu \mathrm{g} / \mathrm{ml})$

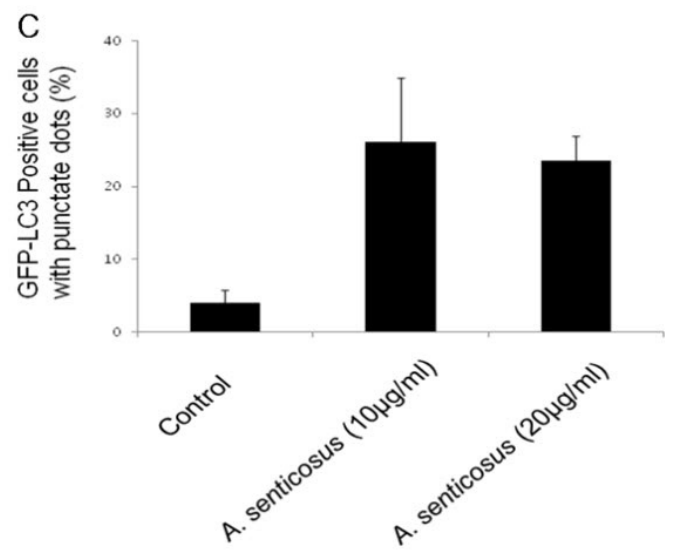

Fig. 3. Induction of autophagy by Siberian ginseng ( $A$ senticosus) extract in HeLa GFP-LC3 cells.

(A) The conversion of GFP-LC3I into GFP-LC3ll in HeLa GFP-LC3 cells treated with concentrations of $A$. senticosus extract for $24 \mathrm{~h}$ was monitored by immunoblot analysis.

(B) The accumulation of GFP protein in autophagosomes in HeLa GFP-LC3 cells after treatment with A senticosus $(50 \mu \mathrm{g} / \mathrm{ml})$ for 24 $\mathrm{h}$ was assessed by fluorescence microscopy.

(C) Autophagy induction after incubation of HeLa GFP-LC3 cells in the presence of $A$ senticosus extract $(10 \mu \mathrm{g} / \mathrm{ml}$ or $20 \mu \mathrm{g} / \mathrm{ml})$ for $24 \mathrm{~h}$ was quantified by counting the number of cells with GFP-LC3 punctate dots. Autophagy induction was quantified as mean $\pm S D$ of combined results from three independent experiments. 
from National Research Foundation of Korea (2012R1A1A2007843) and Kirin Faculty Grant from Daegu Haany University.

\section{Conflict of Interest}

All authors declare no conflict of interest.

\section{References}

1. Wullschleger S, Loewith $\mathrm{R}$, Hall MN. TOR signaling in growth and metabolism. Cell. 2006; 124:471-84.

2. Laplante M, Sabatini DM. mTOR signaling at a glance. J Cell Sci. 2009;122:3589-94.

3. Ikenoue $\mathrm{T}$, Inoki $\mathrm{K}$, Yang $\mathrm{Q}$, Zhou $\mathrm{X}$, Guan KL. Essential function of TORC2 in PKC and Akt turn motif phosphorylation, maturation and signalling. EMBO J. 2008;27:1919-31.

4. Bhaskar PT, Hay N. The two TORCs and Akt. Dev Cell. 2007;12(4):487-502.

5. Hara K, Yonezawa K, Kozlowski MT, Sugimoto $\mathrm{T}$, Andrabi K, Weng QP, et al. Regulation of eIF-4E BP1 phosphorylation by mTOR. J Biol Chem. 1997;272:26457-63.

6. Burnett PE, Barrow RK, Cohen NA, Snyder SH, Sabatini DM. RAFT1 phosphorylation of the translational regulators p70 S6 kinase and 4E-BP1. Proc Natl Acad Sci USA. 1998;95: 1432-7.

7. Khamzina L, Veilleux A, Bergeron S, Marette A. Increased activation of the mammalian target of rapamycin pathway in liver and skeletal muscle of obese rats: possible involvement in obesity-linked insulin resistance. Endocrinology 2005; 146:1473-81.

8. Laplante M, Sabatini DM. mTOR signaling in growth control and disease. Cell. 2012;149: 274-93.

9. Zoncu R, Efeyan A, Sabatini DM. mTOR: from growth signal integration to cancer, diabetes and ageing. Nat Rev Mol Cell Biol. 2011;12(1): 21-35.

10. Harrison DE, Strong R, Sharp ZD, Nelson JF, Astle CM, Flurkey k, et al. Rapamycin fed late in life extends lifespan in genetically heterogeneous mice. Nature. 2009;460(7253):392-5.

11. Eisenberg T, Knauer H, Schauer A, Buttner S, Ruckenstuhl C, Carmona-Gutierrez D, et al. Induction of autophagy by spermidine promotes longevity. Nat Cell Biol. 2009;11(11):1305-14.

12. Metcalfe SM, Canman CE, Milner J, Morris RE, Goldman S, Kastan MB. Rapamycin and p53 act on different pathways to induce G1 arrest in mammalian cells. Oncogene. 1997;15:1635-42.

13. Dowling RJ, Topisirovic I, Fonseca BD, Sonenberg N. Dissecting the role of mTOR: lessons from mTOR inhibitors. Biochim Biophys Acta. 2010;1804(3):433-9.

14. Faivre S, Kroemer G, Raymond E. Current development of mTOR inhibitors as anticancer agents. Nat Rev Drug Discov. 2006;5(8):671-88.

15. Murgia MG, Jordan S, Kahan BD. The side effect profile of sirolimus: a phase I study in quiescent cyclosporine-prednisone-treated renal transplant patients. Kidney Int. 1996;49(1):209-16.

16. Tsang CK, Qi H, Liu LF, Zheng XF. Targeting mammalian target of rapamycin (mTOR) for health and diseases. Drug Discov Today. 2007; 12(3-4):112-24.

17. Hille U, Soergel P, Makowski L, Dork-Bousset T, Hillemanns P. Lymphedema of the breast as a symptom of internal diseases or side effect of mTOR inhibitors. Lymphat Res Biol. 2012;10(2): 63-73.

18. Pilotte AP, Hohos MB, Polson KM, Huftalen TM, Treister N. Managing stomatitis in patients treated with mammalian target of rapamycin inhibitors. Clin J Oncol Nurs. 2011;15(5):E83-9.

19. Skrzypek J, Krause W. Azoospermia in a renal transplant recipient during sirolimus (rapamycin) treatment. Andrologia. 2007;39(5):198-9. 
20. Huang L, Zhao H, Huang B, Zheng C, Peng W, Qin L. Acanthopanax senticosus: review of botany, chemistry and pharmacology. Pharmazie. 2011;66(2):83-97.

21. Zhu HS, Yang XL, Wang LB, Zhao DX, Chen L. Effects of extracts from sporoderm-broken spores of Ganoderma lucidum on HeLa cells. Cell Biol Toxicol. 2000;16:201-6.

22. Mizushima N, Klionsky DJ. Protein turnover via autophagy: implications for metabolism. Annu Rev Nutr. 2007;27:19-40.

23. Suk J, Kwak SS, Lee JH, Choi JH, Lee SH, Lee $\mathrm{DH}$, et al. Alkaline stress-induced autophagy is mediated by mTORC1 inactivation. J Cell Biochem. 2011;112(9):2566-73.

24. Ramirez-Valle F, Badura ML, Braunstein S, Narasimhan M, Schneider RJ. Mitotic raptor promotes mTORC1 activity, G2/M cell cycle progression, and internal ribosome entry site-mediated mRNA translation. Mol Cell Biol. 2010;30(13):3151-64.

25. Sun SY, Rosenberg LM, Wang X, Zhou Z, Yue $\mathrm{P}, \mathrm{Fu} \mathrm{H}$, et al. Activation of Akt and eIF4E survival pathways by rapamycin-mediated mammalian target of rapamycin inhibition. Cancer Res. 2005;65(16):7052-8.

26. Choo AY, Yoon SO, Kim SG, Roux PP, Blenis J. Rapamycin differentially inhibits S6Ks and 4E-BP1 to mediate cell-type-specific repression of mRNA translation. Proc Natl Acad Sci USA. 2008;105(45):17414-9.

27. Breuleux M, Klopfenstein N, Stephan C, Doughty CA, Barys L, Maira SM, et al. Increased AKT S473 phosphorylation after mTORC1 inhibition is rictor dependent and does not predict tumor cell response to PI3K/mTOR inhibition. Mol Cancer Ther. 2009;8(4):742-53.

28. Manning BD, Cantley LC. AKT/PKB signaling: navigating downstream. Cell. 2007;129:1261-74.

29. Watanabe R, Wei L, Huang J. mTOR signaling, function, novel inhibitors, and therapeutic targets. J Nucl Med. 2011;52(4):497-500.

30. Mori $\mathrm{H}$, Inoki $\mathrm{K}$, Masutani $\mathrm{K}$, Wakabayashi $\mathrm{Y}$, Komai K, Nakagawa R, et al. The mTOR pathway is highly activated in diabetic nephropathy and rapamycin has a strong therapeutic potential. Biochem Biophys Res Commun. 2009;384(4): 471-5.

31. Jung $\mathrm{CH}$, Jun $\mathrm{CB}$, Ro SH, Kim YM, Otto NM, Cao J, et al. ULK-Atg13-FIP200 complexes mediate mTOR signaling to the autophagy machinery. Mol Biol Cell. 2009;20(7):1992-2003.

32. Levine B, Kroemer G. Autophagy in the pathogenesis of disease. Cell. 2008;132(1):27-42.

33. Wang X, Proud CG. Nutrient control of TORC1, a cell-cyle regulator. Trends Cell Biol. 2009; 19(6):260-7. 